\title{
Subverting misconceptions about radiation therapy
}

\section{To the Editor:}

More than a century after the discovery of ionizing radiation, its pleomorphic effects on living organisms continue to puzzle and inspire investigations on how to optimize this powerful therapeutic tool. The recent publication by Price et al. investigates the effect of whole-body irradiation on radiation-resistant Langerhans cells (LCs) and their migration to lymph nodes to elicit the generation of regulatory $\mathrm{T}$ cells $\left(\mathrm{T}_{\text {reg }} \text { cells }\right)^{1}$. The authors ascribe the radiation resistance of LCs to heightened activation of the cyclin-dependent kinase inhibitor CDKN1A (p21), a stalwart mechanism of protection from radiation in many normal and malignant cells. Cell-cycle arrest mediated by $\mathrm{p} 21$ extends the opportunity for a cell to execute the DNA-damage response and repair and thereby avoid deletion by apoptosis.

The mechanism described builds on preexisting evidence of diminished immunosurveillance of irradiated normal skin ${ }^{2}$ and introduces an immunological dimension to the role of radiation as a carcinogen ${ }^{3}$. The authors speculate that the peculiar resistance of LCs to radiation and their induction of $\mathrm{T}_{\text {reg }}$ cells might have evolved as a mechanism to preclude autoimmunity toward the skin, an organ constantly exposed to damage from ultraviolet radiation. Interestingly, among atomic-bomb survivors exposed to total-body radiation, the excess relative risk of skin cancer was $15,5.7$ or 1.3 as a function of age with exposure at an age of 0-9, $10-19$ or $20-39$ years, respectively ${ }^{4}$. This agedependent effect is intriguing from an immunological point of view, since an opposite trend would be expected on the basis of diminishing immunocompetence with age.

However, the generalization of these findings to clinical radiotherapy is invalid.

Experimental mice received total-body irradiation (TBI) in large doses (6 or 12 Gy, near and exceeding, respectively, the dose lethal to

Price, Idoyaga and Merad reply:

A recent article from our group reported on an underlying mechanism of resistance to depletion by ionizing irradiation that is used by LCs, a unique population of dendritic cells
$50 \%$ of C57BL/ 6 mice) before being challenged by subcutaneous injections of B16 melanoma tumor cells. Irradiated mice developed larger tumors than their unirradiated control counterparts did shortly after TBI treatment (within 12-24 h), but the effect was abolished when mice were inoculated 5 weeks after TBI.

As Price et al. acknowledge in the discussion of their findings ${ }^{1}$, the use of TBI in these experiments has little in common with the usual clinical practice of radiotherapy, in which localized radiation is delivered to established tumors, in a highly targeted fashion, with much effort expended to avoid normal tissue through the strategic use of fractions of much lower dose administered over time. Extensive experience in treating skin cancer with single-modality radiotherapy has demonstrated lasting tumor control in approximately $90 \%$ of basal cell carcinomas and $80 \%$ of squamous cell carcinomas ${ }^{5}$. Radiation therapy has maintained its solid role in the therapeutic arsenal for the treatment skin cancer since the $1900 s^{6}$, a fact difficult to reconcile with the conclusions of Price et al. ${ }^{1}$. Unfortunately, misinterpretation of this paper as evidence for a general immunosuppressive action of radiotherapy is already being delivered to the public (http://medicalxpress.com/ news/2015-09-doctors-caution-radiotherapyskin-cancer.html).

Thus, the work of Price et al. ${ }^{1}$ needs to be considered in the context of current research delineating how localized radiotherapy of cancer can have both pro-immunogenic effects and immunosuppressive effects. The identification of critical cross-talk between radiation-induced signals and the immune system of cancer carriers offers the opportunity to both optimize the clinical use of radiotherapy and enhance the effects of cancer immunotherapies ${ }^{7}$. This rationale has inspired ongoing therapeutic investigations that combine immunotherapy agents to correct the immuno- suppressive effects of radiation and/or enhance its immune system-promoting effects. For example, clinical radiotherapy can be successfully combined with blockade of immunological checkpoints that counteracts a radiation-induced increase in $\mathrm{T}_{\text {reg }}$ cells ${ }^{8}$. Moreover, evidence is emerging that local radiation therapy can convert a tumor into an individualized cancer vaccine in a setting of otherwise ineffective immunotherapy and can work in concert with immunotherapy to control the primary tumor and metastasis outside the radiation field ${ }^{9,10}$.

\section{COMPETING FINANCIAL INTERESTS}

The authors declare no competing financial interests.

Silvia C Formenti ${ }^{1}$, Sandra Demaria ${ }^{1}$, Mary Helen Barcellos-Hoff ${ }^{2}$ \& William H McBride ${ }^{3}$

${ }^{1}$ Department of Radiation Oncology, Weill Cornell Medical College, New York, New York, USA. ${ }^{2}$ Department of Radiation Oncology, University of California, San Francisco, San Francisco, California, USA. ${ }^{3}$ Division of Molecular and Cellular Oncology, University of California, Los Angeles, Los Angeles, California, USA.

e-mail:wmcbride@mednet.ucla.edu or formenti@cornell.edu

1. Price, J.G. et al. Nat. Immunol. 16, 1060-1068 (2015).

2. Cummings, R.J. et al. J. Immunol. 189, 4247-4257 (2012).

3. Nguyen, D.H. et al. Cancer Cell 19, 640-651 (2011).

4. Sugiyama, H. et al. Radiat. Res. 181, 531-539 (2014).

5. Locke, J., Karimpour, S., Young, G., Lockett, M.A \& Perez, C.A. Int. J. Radiat. Oncol. Biol. Phys. 51, 748-755 (2001).

6. Bath-Hextall, F.J., Perkins, W., Bong, J. \& Williams, H.C. Cochrane Database Syst. Rev. 1, CD003412 (2007).

7. Demaria, S., Bhardwaj, N., McBride, W.H. \& Formenti, S.C. Int. J. Radiat. Oncol. Biol. Phys. 63, 655-666 (2005).

8. Golden, E.B. et al. Immunol. Res. 1, 365-372 (2013).

9. Brody, J.D. et al. J. Clin. Oncol. 28, 4324-4332 (2010).

10. Golden, E.B. et al. Lancet Oncol. 16, 795-803 (2015). that reside in the epidermis ${ }^{1}$. Formenti et al. raise one major objection to our report: that our mouse model system bears little resemblance to clinical radiotherapy. In our tumorchallenge experiments, we administered TBI at a dose of 6 Gy to mice that we subsequently challenged subcutaneously with B16 cells. As stated in our initial report, our goal was twofold. First, we sought to determine the effect of conditioning-radiation therapy on the local 